\title{
Compression with considerable sidelobe suppression effect in weather radar
}

\author{
Haijiang Wang ${ }^{1,2^{*}}$, Zhao Shi ${ }^{1,2}$ and Jianxin $\mathrm{He}^{1,2}$
}

\begin{abstract}
Pulse compression is a classical topic. Because of its function in resolution enhancement, pulse compression technology has been applied in many kinds of radar such as pulse Doppler weather radar. In pulse compression, sidelobe suppression plays a key role for reducing clutter. In this article, a combination of two sidelobe suppression techniques for pulse compression is proposed. Simulation results show that the combination of the two techniques has better sidelobe suppression effectiveness.
\end{abstract}

Keywords: Pulse compression, Sidelobe suppression, Weather radar

\section{Introduction}

Radar, serving as a kind of method for detecting, has wide-range application areas such as military, aviation, geosciences, and so on. Pulse Doppler weather radar is an important device to detect clouds, wind fields, and precipitation. So, the resolution is very important for the forecasting of weather phenomena.

Pulse compression is useful for weather radar to increase the average transmitted power by transmitting a longer pulse but without reducing the range resolution of the radar. In general, peak power of solid-state transmitter or millimeter-wave klystron is not sufficient. Pulse compression is required to achieve the desired system sensitivity. Different coding schemes include linear frequency modulation (LFM), nonlinear FM. Frequencymodulated signals are characterized by their time bandwidth (TB) product which represents the ability to multiple signal-to-noise ratio (SNR).

The foundation of pulse compression is matched filtering [1]. In time domain, matched filtering is equivalent to the autocorrelation of the input signal. In frequency domain, the transfer function of the matched filter is the complex conjugate of the input signal's spectrum. An implementation schedule of the matched filter in frequency domain is shown in Figure 1 [1].

\footnotetext{
* Correspondence: whj@cuit.edu.cn

${ }^{1}$ College of Electronic Engineering, Chengdu University of Information

Technology, Chengdu, China

${ }^{2} \mathrm{CMA}$, Key Laboratory of Atmospheric Sounding, Chengdu, China
}

For a radar pulse signal, after compression, the narrower pulse mainlobe is always accompanied by higher sidelobes. So, all pulse compression radars suffer from range side lobes which cause energy from strong reflections to leak into adjacent range cells. High suppression of side lobes is not required in some other nonmeteorological radar, but is important for meteorological radar, because weather phenomena can have significant reflectivity gradients, and ground clutter echo can be between 35 and $55 \mathrm{~dB}$ much larger than medium rain. Furthermore, the side lobes of strong signal will falsely be recognized as an existence of small target. Therefore, range side lobes must be suppressed by a large amount to prevent contamination in adjacent range cells.

In this article, compression experiments are conducted on an LFM sample signal extracted from a period of echo of a weather radar. In these experiments, some sidelobe suppression algorithms are used. The algorithms include multiplying window in frequency domain, phase distortion, and frequency modification.

In Section 2, the data are analyzed and compression basing on matched filtering and windowed matched filtering are carried out. In Section 3, spectrum modification technique and phase predistortion technique are utilized, respectively, and the effects are analyzed. Then, the two techniques are combined in Section 4. Experiments are conducted on the real weather echo and the effects are compared. Section 5 is the conclusion. 


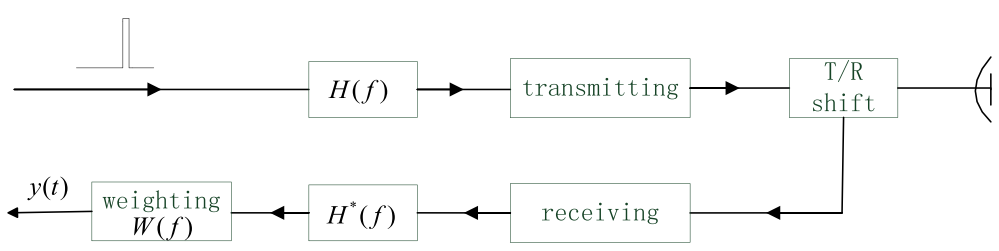

Figure 1 Implementation of matched filter by conjugate filters pair.

\section{LFM pulse and matched filtering compression}

A period of weather echo of a pulse Doppler radar is shown in Figure 2. The sample rate of the echo is 4.8 $\mathrm{MHz}$. The pulse width is $33.3 \mu \mathrm{s}$ and the spectrum width after LFM is $2 \mathrm{MHz}$.

A section which is a whole LFM pulse signal is intercepted from the echo above. This LFM pulse, shown in Figure 3, can be taken as the sample signal to do pulse compression. Its amplitude spectrum is shown in Figure 4.

To analyze the frequency modulation quality of the pulse in Figure 3, we extract the phase information and unwrap it. The phases before and after unwrapping are shown in Figures 5 and 6, respectively.

Quadratic curve fitting is done on the phase, the fitting result is illustrated in Figure 7.

We found that the phase and the fitting curve superpose each other. This indicates that the linearity of the frequency is good and the pulse is a suitable LFM sample.

Matched filtering [1] is carried out on the LFM pulse directly and the compression result is shown in Figure 8.

The amplitude of the first sidelobe is $-17.4827 \mathrm{~dB}$ and the compression ratio is about 83.3.
A sidelobe of $-17.4827 \mathrm{~dB}$ is not satisfying for most target detecting, because there will be serious range ambition if the sidelobe is too high.

During matching filtering, multiplying a window in the frequency domain can suppress the sidelobe to a certain level. But in general, the lower sidelobe is often accompanied by a wider mainlobe after windowed matched filtering.

The general formation of a weighting window can be expressed as follows [2]:

$$
H(f)=K+(1-K) \cos ^{n}\left(\frac{\pi f}{B}\right)
$$

When $K=0.08$ and $n=2$, the weighting function is a Hamming window; when $K=0.333$ and $n=2$, the function is a 3:1 taper weighting, when $K=0$ and $n=2,3,4$, respectively, the functions are cosine square, cosine cube, and cosine quartic weighting.

Weighting the LFM pulse in frequency domain with a Hamming window, the window and the compressed pulse after matched filtering are shown in Figures 9 and 10.

It can be seen from Figure 10 that the sidelobe is suppressed to the level of $-37 \mathrm{~dB}$. From theoretical

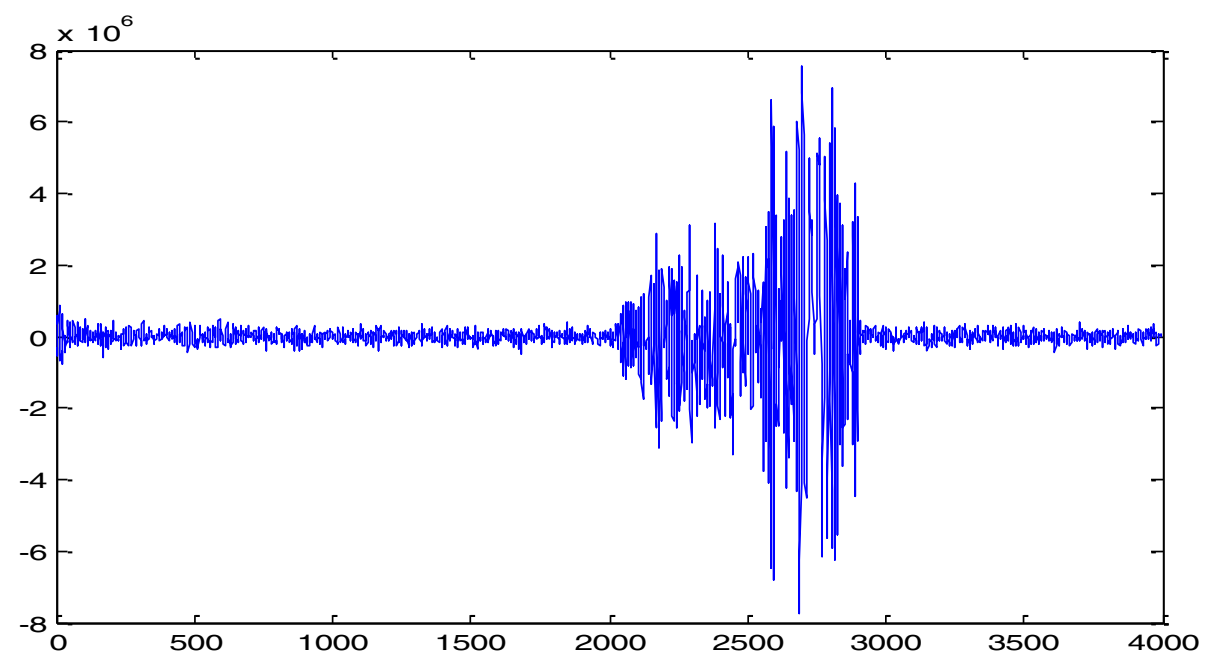

Figure $2 \mathrm{~A}$ period of weather echo of a pulse Doppler radar. 


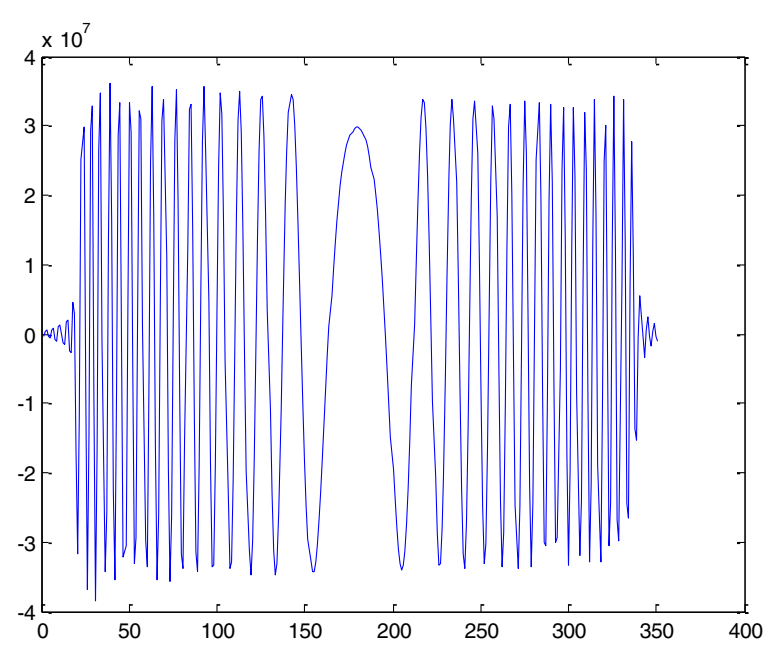

Figure 3 The LFM pulse intercept from the echo.

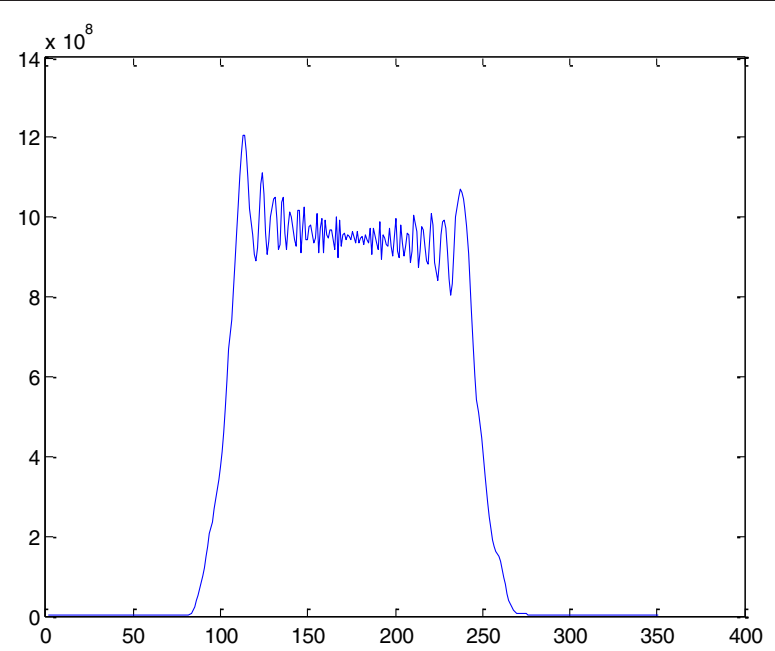

Figure 4 The spectrum of the LFM pulse.

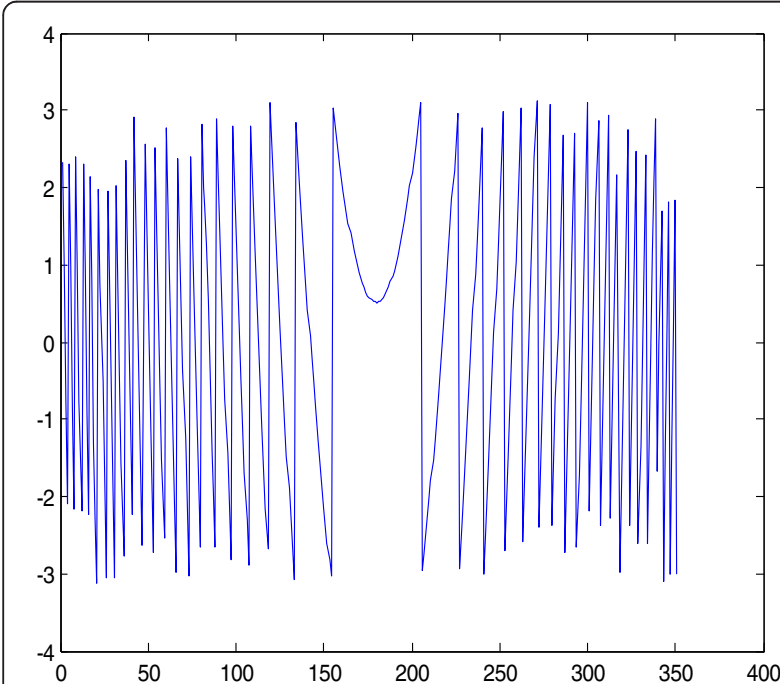

Figure 5 The phase before unwrapping.

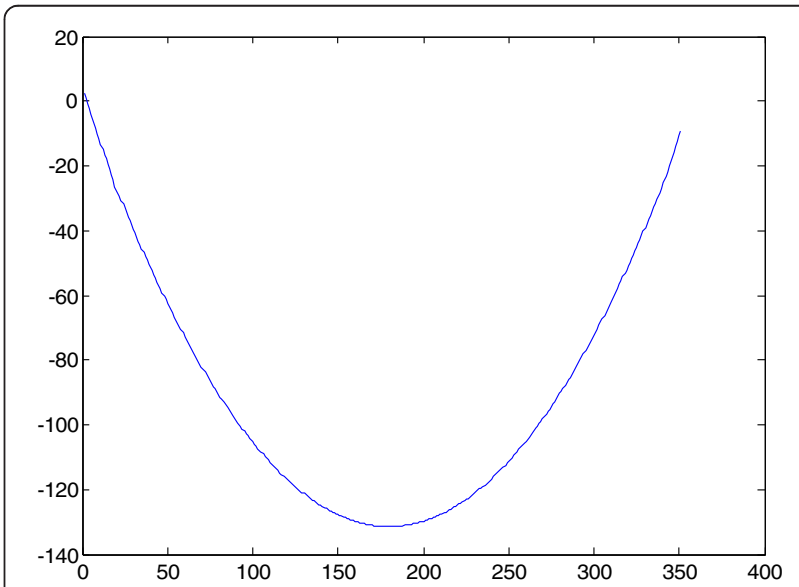

Figure 6 The phase after unwrapping. 


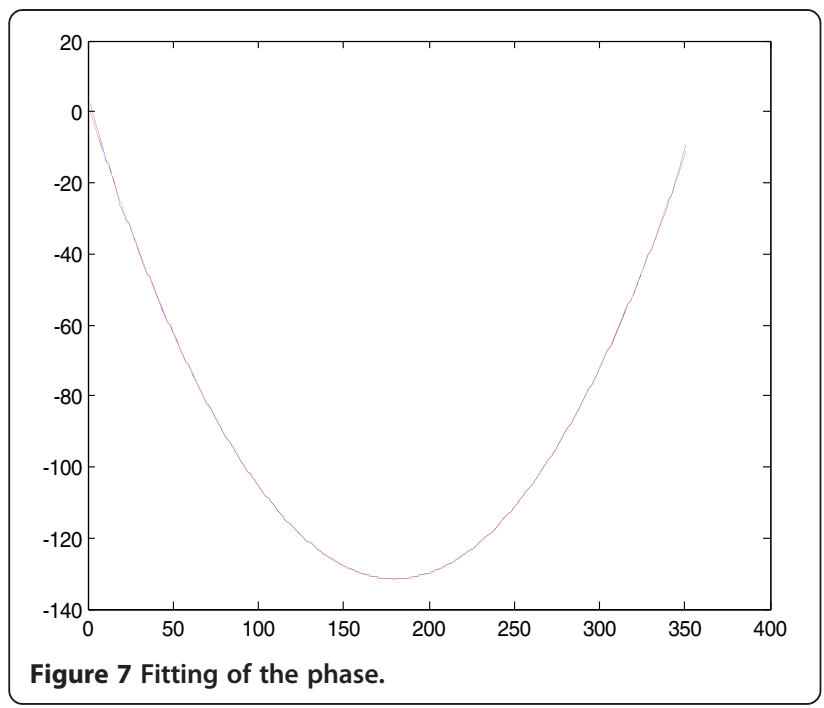

analysis we can educe that the mainlobe broaden to 1.47 times of the one before windowing.

For Doppler weather radar, to enhance the accuracy of weather target detecting, a lower sidelobe is needed. So, further improvements of pulse compression effects are demanded.

\section{Sidelobe suppression by phase predistortion and spectrum modification}

For an LFM signal with TB product, the cubic phase predistortion technique can be used to suppress the sidelobe $[3,4]$. The signal with little TB product has large ripples in its spectrum band, so widowing is not satisfying for sidelobe suppression. In this situation, the sidelobe suppression can be achieved by suppressing the ripples in band through phase predistortion. This method is easy to implement with surface acoustic wave (SAW) technique.

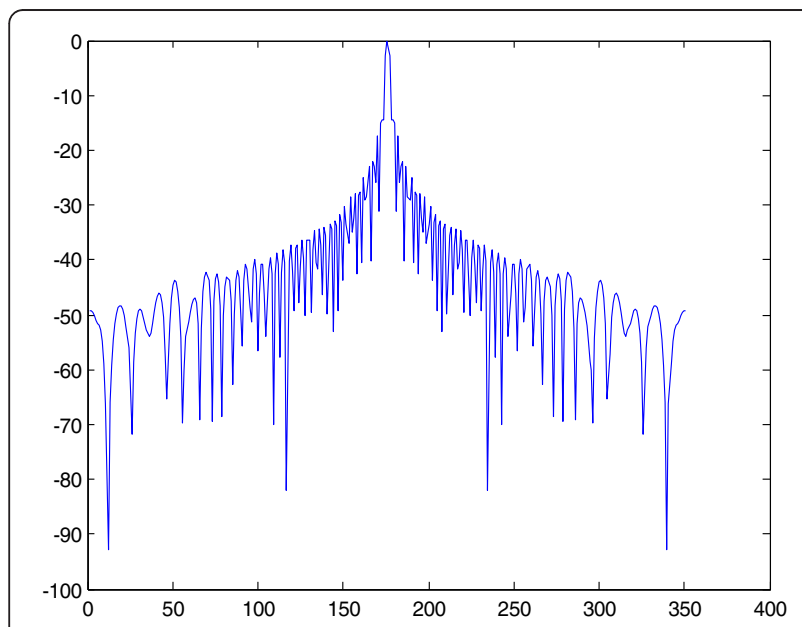

Figure 8 Compression result by matched filtering.

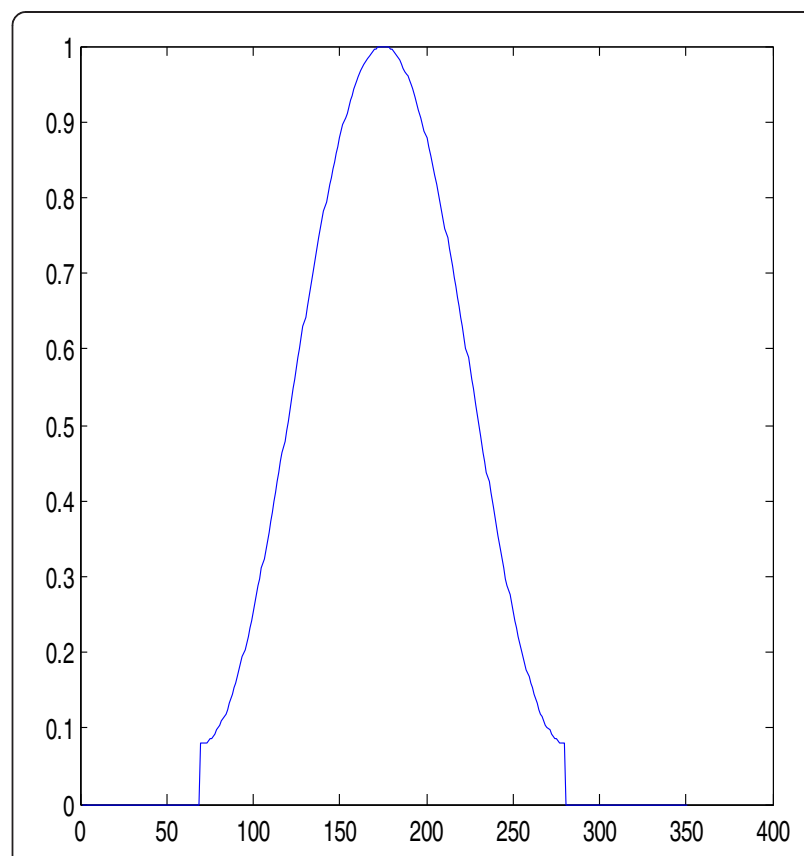

Figure 9 The Hamming window.

Suppose the complex expression of the LMF signal to carry out phase predistortion on is

$$
\begin{aligned}
& s(t)=\exp \left[j 2 \pi\left(f_{0} t+\frac{B}{2 T} \cdot t^{2}+\phi(t)\right)\right], \\
& -T / 2-\Delta T \leq t \leq T / 2+\Delta T
\end{aligned}
$$

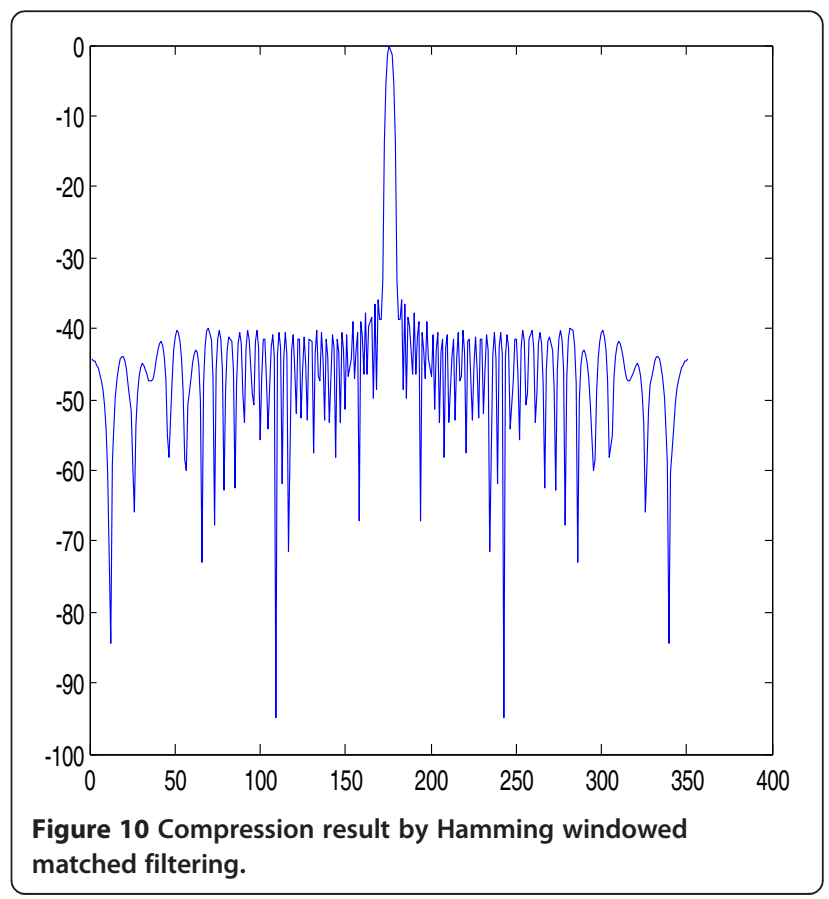




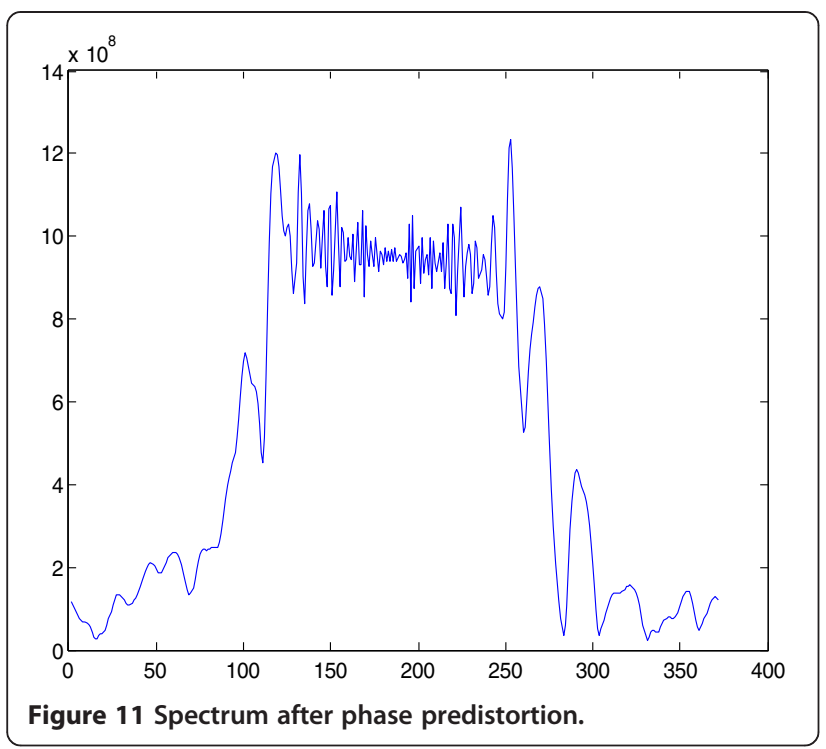

where $f_{0}$ is the central frequency and $B$ is the bandwidth. And the duration of the pulse is $T$. Then the additional phase item can be

$$
\phi(t)=\left\{\begin{array}{cl}
\frac{\Delta B}{3 \Delta T^{2}} \cdot(-t-T / 2)^{3}, & -T / 2-\Delta T \leq t<-T / 2 \\
\frac{\Delta B}{3 \Delta T^{2}} \cdot(t-T / 2)^{3}, & T / 2<t \leq T / 2+\Delta T \\
0, & \text { elsewhere }
\end{array}\right.
$$

where $\Delta T=1 / B$ and $\Delta B=0.75 B[3]$.

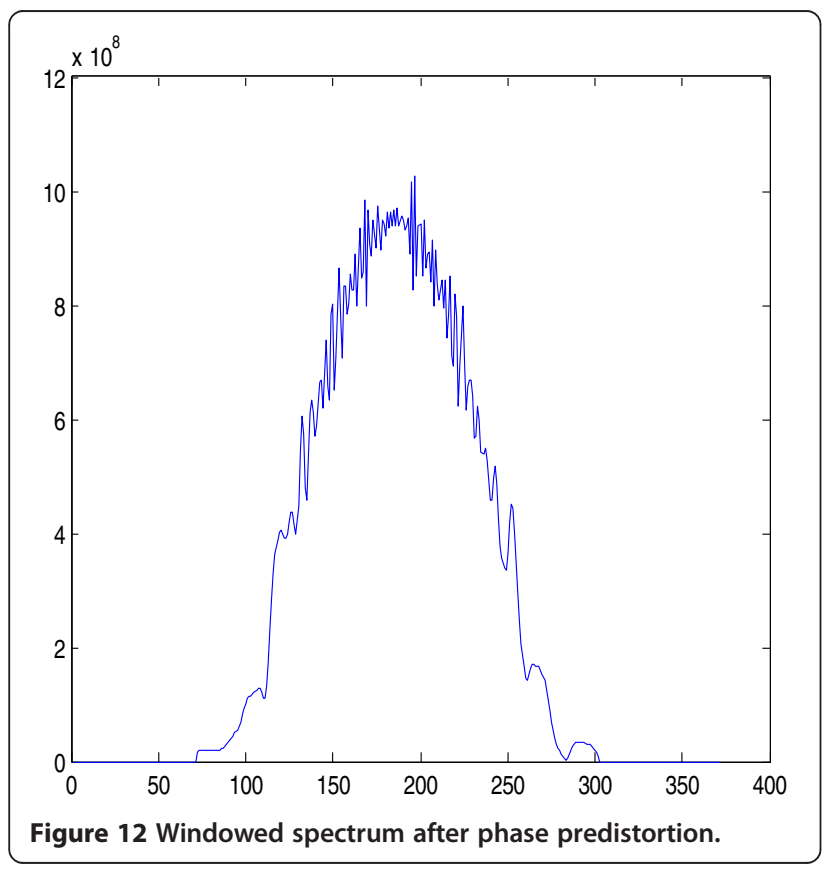

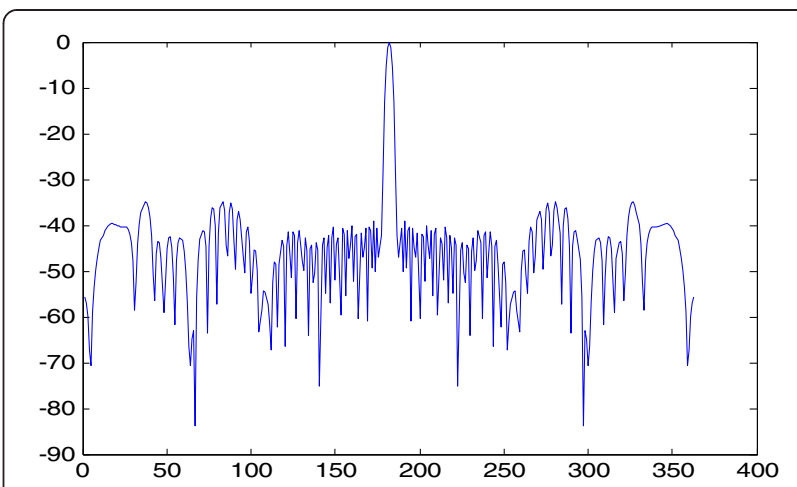

Figure 13 Compression result by windowed matched filtering after phase predistortion.

The spectrum of the pulse after phase predistortion is shown in Figure 11 and after multiplying a Hamming window, the predistorted spectrum is illustrated as shown in Figure 12.

After matched filtering, the effect of pulse compression is shown in Figure 13.

Figure 13 demonstrated that the sidelobes neighboring to the mainlobe are suppressed well $(3-4 \mathrm{~dB})$ through matched filtering after phase-predistortion. But in the positions away from the mainlobe, there are some sidelobe hunches and this may bring range ambiguity of two targets far away from each other.

In ideal situation, the output of the matched filter for an LFM signal has rectangular spectrum. After weighting, the rectangular spectrum becomes a certain window. But when the TB product of the LFM signal is small, its ripples in band are large, so the weighting has little effects for ripple suppression in band. In this case, spectrum modification technique can be resorted to make the processed spectrum approaches ideal window function mostly $[5,6]$ and to enhance the main-to-side lobe ratio.

Spectrum modification can be implemented by modifying the transfer function of the matched filter. Suppose

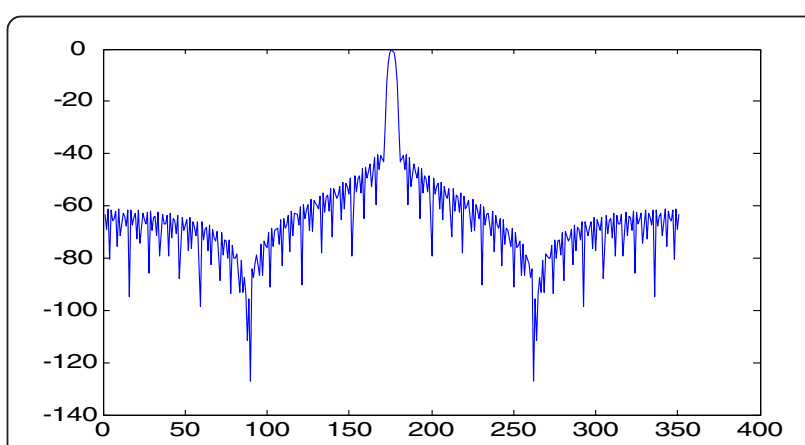

Figure 14 Compression result by windowed matched filtering after spectrum modification. 
the spectrum of the LFM signal is $U(f)$ and the transfer function is $H(f)$, to make the output of the matched filtering to be a rectangular, it is needed that:

$$
U(f) H(f)=I(f) I^{*}(f), I(f)=\operatorname{rect}(f / B)
$$

The modified transfer function of the matched filter is

$$
H(f)=U^{*}(f)\left[I(f) I^{*}(f) /|U(f)|^{2}\right]
$$

The pulse compression result after using spectrum modification is shown in Figure 14.

From Figure 14, it can be seen that spectrum modification technique has good suppression effect for the sidelobes neighboring the mainlobe. What is the most important, this technique bring a great advantage that suppress the sidelobes far away from the mainlobe to the level under $-62 \mathrm{~dB}$.

It is stressed that both phase predistortion and spectrum modification did not spread the mainlobe apparently.

\section{Combination of phase predistortion and spectrum modification}

From the simulation results in Section 3, we can find that both phase predistortion and spectrum modification have the ability of sidelobe suppression. But phase predistortion can bring hunches far away from the mainlobe and spectrum modification has good effect of suppression to the sidelobes in the distance. So, we predict that the combination of these two techniques will bring better pulse compression performance. The

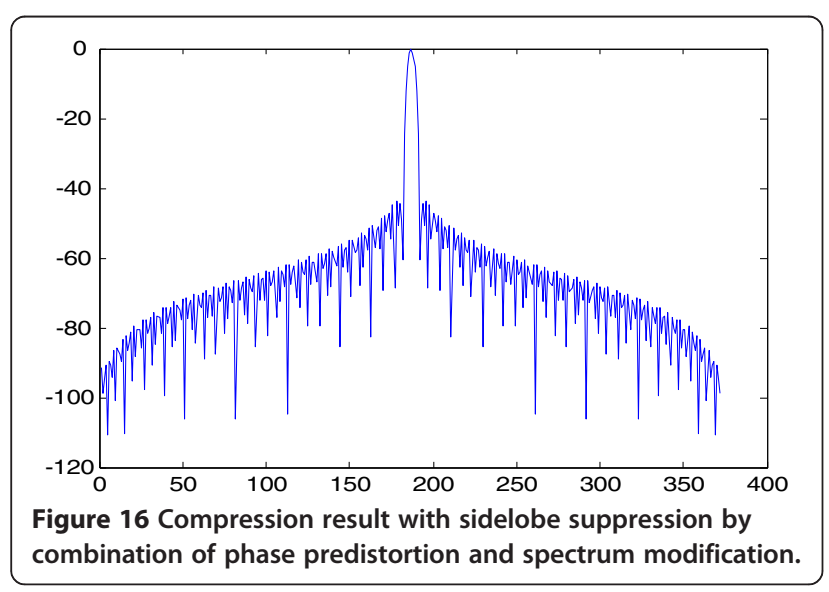

two techniques are used sequentially as shown in Figure 15.

Figure 16 is the result of the simulation utilizing two techniques sequentially.

From Figure 16, it can be seen that

- The first sidelobe is suppressed to the level under $45 \mathrm{~dB}$, and the sidelobes attenuate quickly when getting far away from the mainlobe.

- The envelope of the sidelobes is monotonically decreasing.

- The mainlobe does not spread apparently comparing with the result only using weighting function.

In order to compare the effectiveness for pulse compression and sidelobe suppression of the several methods collectively, the data are listed in Table 1.

The whole sky weather echoes before and after pulse compression are shown in Figure 17.

It can be seen from Figure 17 that

- The resolution of weather radar echo is very poor without pulse compression.

- Phase predistortion and spectrum modification can bring comparative resolution enhancement through sidelobe suppression. But spectrum modification technique brings some false target echo in the upper left corner of the reflectivity section.

- The combination of phase predistortion and spectrum modification has the best resolution.

- Furthermore, it is evaluated to statistics the weather echoes SNR of three methods. The mean SNR value of compression with phase predistortion is $0.044 \mathrm{~dB}$ less than compression with phase predistortion and spectrum modification, the variance value is 0.4528 $\mathrm{dB}$. The mean SNR value of compression with

Table 1 Effectiveness of pulse compression and sidelobe suppression

\begin{tabular}{llll}
\hline Methods & $\begin{array}{l}\text { Mainlobe } \\
\text { compression } \\
\text { ratio }\end{array}$ & $\begin{array}{l}\text { Sidelobe } \\
\text { suppression } \\
\text { (dB) }\end{array}$ & $\begin{array}{l}\text { Sidelobe } \\
\text { attenuation } \\
\text { speed }\end{array}$ \\
\hline $\begin{array}{l}\text { No sidelobe } \\
\text { suppression }\end{array}$ & $1 / 833$ & -17.5 & Fast \\
$\begin{array}{l}\text { Hamming } \\
\text { window only }\end{array}$ & $1 / 567$ & -37.0 & Slow \\
\hline
\end{tabular}



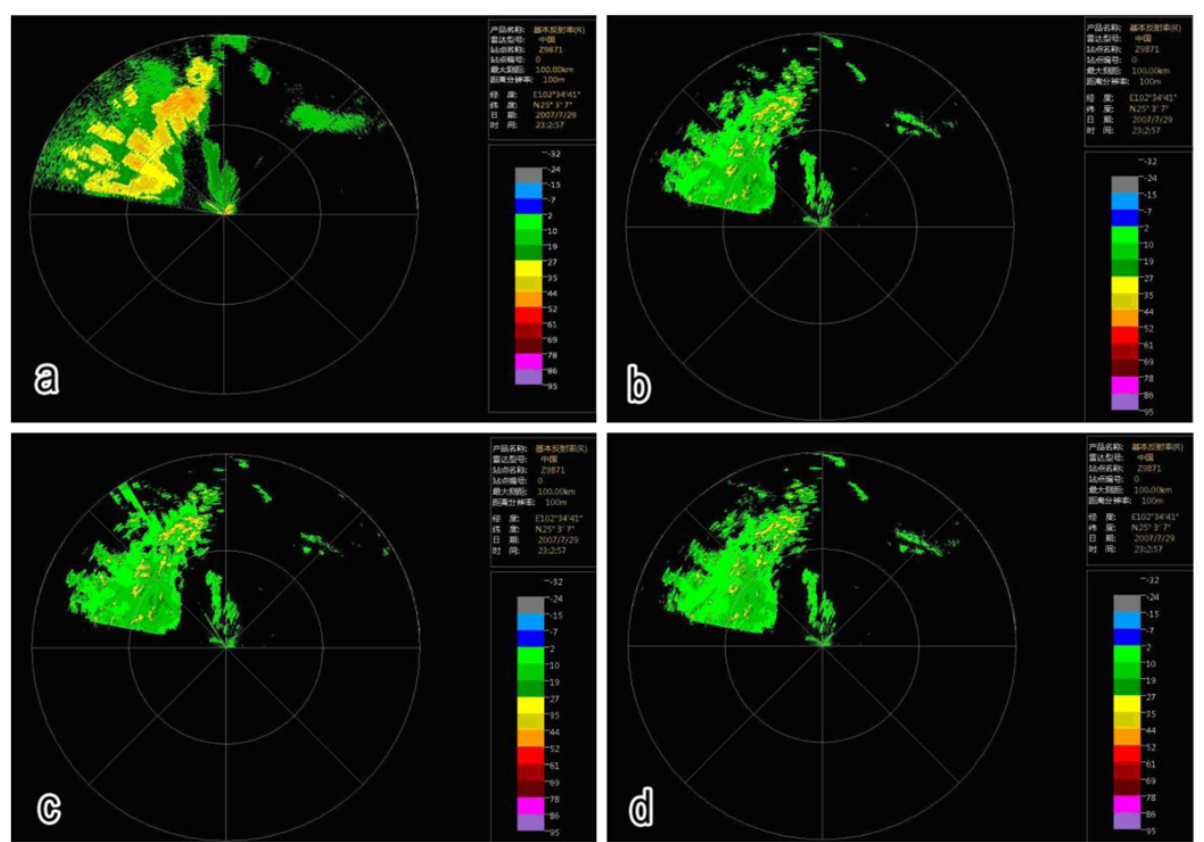

Figure 17 The whole sky weather radar echoes. (a) Echo with no pulse compression, (b) Echo after compression with phase predistortion, (c) Echo after compression with spectrum modification, (d) Echo after compression with phase predistortion and spectrum modification.

spectrum modification is $0.017 \mathrm{~dB}$ less than compression with phase predistortion and spectrum modification, the variance value is $0.2664 \mathrm{~dB}$.

- Figure 18 shows the SNR statistical comparison between phase predistortion method and combined method of phase predistortion and spectrum modification.

- Figure 19 shows the SNR statistical comparison between spectrum modification and combined method of phase predistortion and spectrum modification.

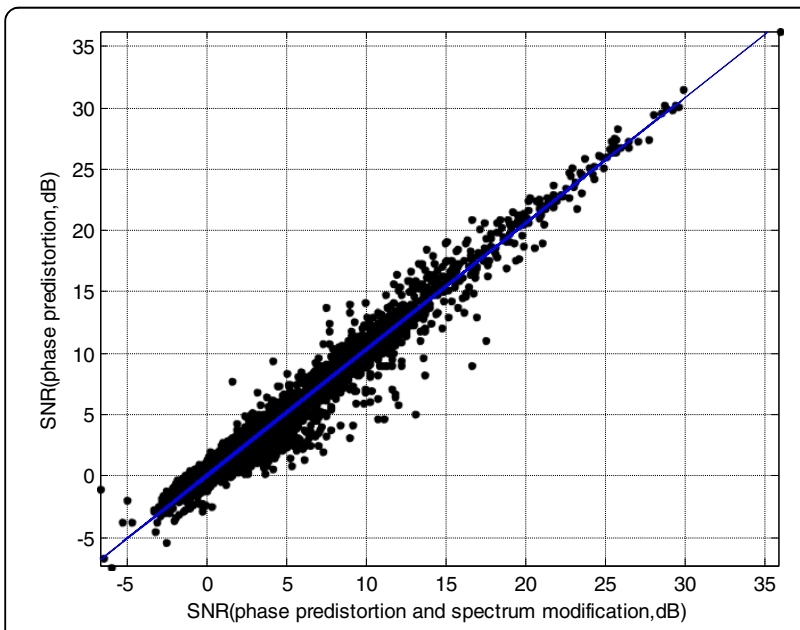

Figure 18 SNR statistical comparison between phase predistortion method and combined method of phase predistortion and spectrum modification.

\section{Conclusion}

Sidelobe suppression is an important part in pulse compression. From the simulations and application on weather radar, it can be seen that the combination of phase predistortion and spectrum modification technique has good sidelobe suppression performance. In some applications involving hardware implementation and realtime processing, the SAW device can be used to carry out impulse compression [7,8]. Resolution enhancing is important for both weather radar and other kinds of radar, such as the imaging radar $[9,10]$, so it is still a

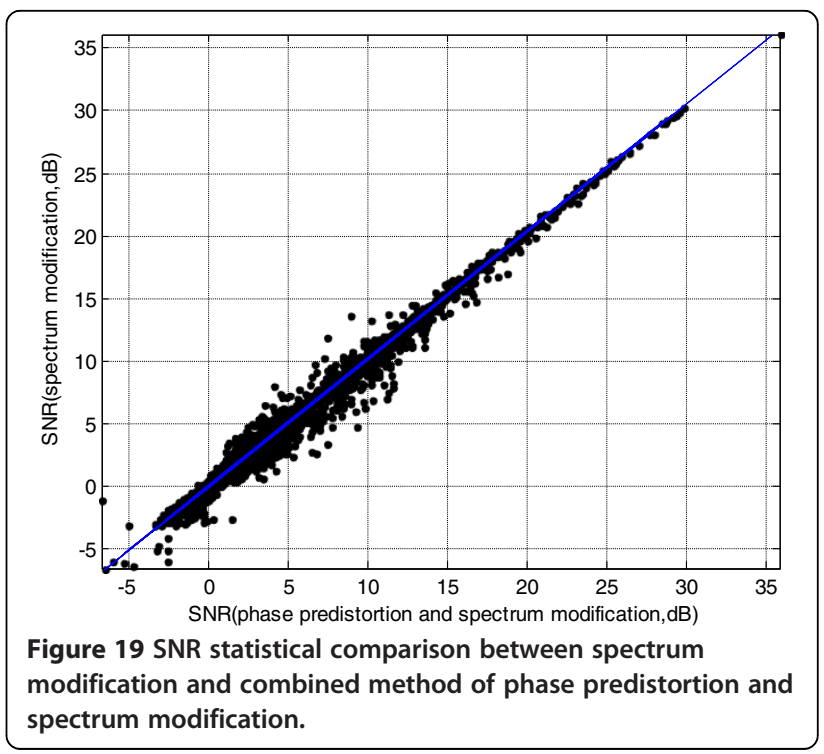


hotspot for research. The methods development by this article can be used in other kinds of radar with pulse compression systems.

\section{Competing interests}

The authors declare that they have no competing interests.

Received: 11 December 2012 Accepted: 26 February 2013

Published: 4 April 2013

\section{References}

1. M Lin, Y Ke, Theory of Radar Signal (National Defense Industry Press, Beijing, 1984), pp. 87-203

2. CE Cook, M Bernfeld, J Paolillo, CA Palmier, Matched filtering, pulse compression and waveform design. Microwave. J. 8, 73-81 (1965)

3. CE Cook, J Paolillo, A pulse compression predistortion function for efficient sidelobe reduction in a high-power radar. Proc. IEEE 52, 377-389 (1964)

4. M Kowatsch, Suppression of sidelobes in rectangular linear FM pulse compression radar. Proc. IEEE 70(3), 308-309 (1982)

5. $Y L v, J$ Xiang, $F$ Chen, A method for reducing range-sidelobes of linear frequency-modulated pulse compression signals. J. UEST China 22(4), 344-349 (1993)

6. B Yang, J Wu, A range sidelobe reduction technique based on modifications to signal spectrum. Syst. Eng. Electron. 22(9), 90-93 (2000)

7. Z Xianmin, X Jinlin, W Hongtai, X Qing, SAW pulse compression systems with lower sidelobes, in Microwave Conference Proceedings, APMC'97, vol. 2 (, Hongkong, 1997), pp. 833-835

8. JW Arthur, Modern SAW-based pulse compression systems for radar applications. I. SAW matched filters. Electron. Commun. Eng. 7(6), 236-246 (1995)

9. J Li, Y Pi, X Yang, Micro-doppler signature feature analysis in Terahertz band. J. Infrared Millim. TE 31(3), 319-328 (2010)

10. J Xu, Y Pi, Z Cao, Bayesian compressive sensing in synthetic aperture radar imaging. IET Radar Sonar Navigat. 6(1), 2-8 (2012)

\section{Submit your manuscript to a SpringerOpen ${ }^{\circ}$ journal and benefit from:}

- Convenient online submission

- Rigorous peer review

- Immediate publication on acceptance

- Open access: articles freely available online

- High visibility within the field

- Retaining the copyright to your article 